\title{
Microwave Synthesis of Silver Sulfide and Silver Nanoparticles: Light and Time Influence
}

\author{
David Magalhães Sousa,* William Chiappim, Joaquim P. Leitão, João Carlos Lima, and Isabel Ferreira
}

Cite This: ACS Omega 2020, 5, 12877-12881

Read Online

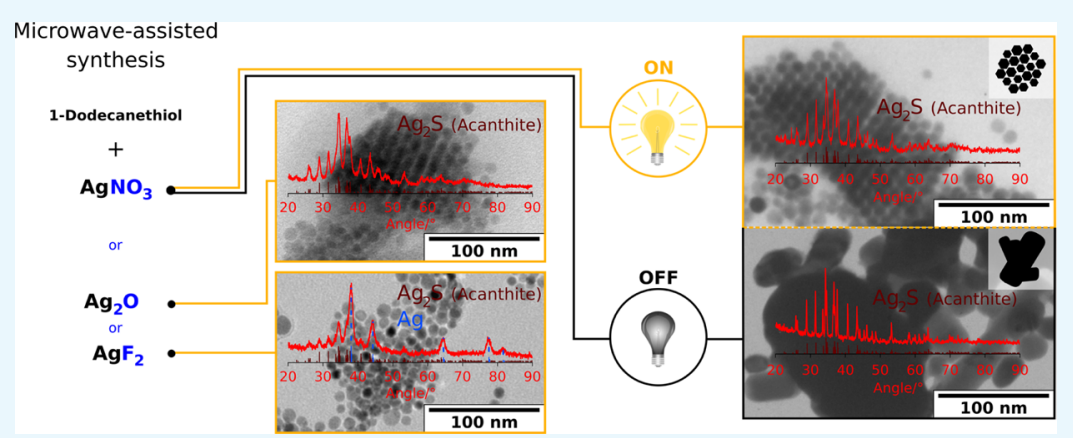

ABSTRACT: Silver sulfide $\left(\mathrm{Ag}_{2} \mathrm{~S}\right)$ is a low band gap material, which absorbs near-infrared light and is of great importance in areas such as nanotechnology and biomedicine. We report the influence of the starting reagents, synthesis time, and light radiation on the geometry and size of silver sulfide nanoparticles and on the fraction of metallic Ag obtained in a microwave reactor. The X-ray diffraction diffractograms confirmed that $\mathrm{Ag}_{2} \mathrm{~S}$ is the main product if the reaction's precursor contains silver in the oxidation state of +1 and mostly metallic silver $\left(\mathrm{Ag}^{\circ}\right)$ when it is +2 . Small nanoparticles $(\sim 6 \mathrm{~nm})$ of spherical geometry are present in the transmission electron microscopy images for the synthesis performed with the lamp light ON, while with the light switched OFF, wider and hundreds of nanometers longer particles are observed. This discriminative effect occurs with shorter synthesis time duration $(<10$ $\mathrm{min}$ ) but when the time of reaction is extended, the particles coalesce for both light and dark conditions. Overall, it was observed by photoluminescence that crystalline $\mathrm{Ag}$ and $\mathrm{Ag}_{2} \mathrm{~S} 4-8 \mathrm{~nm}$ nanoparticles obtained in $15 \mathrm{~min}$ and light irradiation during synthesis have a clear relative increase of the radiative recombination channels of the charged carriers, which are typical of materials characterized by the involvement of low density of states inside the band gap.

\section{INTRODUCTION}

Among different silver compounds, both pure $\mathrm{Ag}_{2} \mathrm{~S}$ nanoparticles and $\mathrm{Ag}_{2} \mathrm{~S}$ nanocomposites proved to be suitable for optoelectronics such as photovoltaic devices, ${ }^{1,2}$ because of their low band gap.

The photosensitivity of silver compounds has been known for decades and given the recent interest in silver sulfide nanoparticles' synthesis and applications, the influence of light during synthesis is scarcely explored, although effects of light irradiation on the stability of $\mathrm{Ag}_{2} \mathrm{~S}$ nanoparticles have been investigated in recent years. ${ }^{3,4}$ Previous reports state that visible light can fragment silver nanoparticles, ${ }^{5}$ and in the presence of both light and organic reducing agents, a photoinduced reduction of the silver salt is observed, resulting in spherical silver nanocrystals. ${ }^{6,7}$ Jin et al. demonstrated a photoinduced method to convert silver nanospheres into triangular nanoprisms. ${ }^{8}$ Visible light promotes a photocatalytic process leading to the formation of $\mathrm{Ag}^{+}$ions at the initial stage, and the subsequent reduction promotes the growth in preferential directions. By observing the role of each reagent during the synthesis of the silver nanoprisms under irradiation,
Xue et al. proposed that the growth of silver nanoprisms is also regulated by a silver redox cycle. Silver cations are reduced by citrate on the surface of pre-existing silver particles, while the smaller ones are oxidatively digested by oxygen. The results were supported by the fact that no geometry changes are observed when the silver seeds are irradiated under nitrogen, and that high silver concentrations or low light intensity also leads to a lower prism yield. ${ }^{9}$ In summary, in the presence of oxygen, water, light, and a soft reducing agent such as the citrate anion, a redox cycle of $\mathrm{Ag}^{\circ} / \mathrm{Ag}^{+}$exist that can be explored to achieve distinct particle geometries.

A study of the impact of the conditions, including light on the morphology of $\mathrm{Ag}_{2} \mathrm{~S}$ nanoparticles is the aim of the present work. For the synthesis of silver sulfide, the sulfur source 1-

Received: February 13, 2020

Accepted: March 27, 2020

Published: May 29, 2020 
dodecanethiol was chosen based on the previous studies. It is a mild reducing agent and releases the sulfide anion at high temperatures, ensuring that small silver sulfide nanoparticles are obtained. ${ }^{10}$ The photochemical conversion of metallic Ag nanoparticles into $\mathrm{Ag}_{2} \mathrm{~S}$ quantum dots $(2 \mathrm{~nm})$ was recently and first demonstrated by $\mathrm{Cao}$ et al. ${ }^{11}$ by introducing 4-tertbutylbenzene-thiol in the nonpolar solvent containing metallic $\mathrm{Ag}$ nanoparticles at $10{ }^{\circ} \mathrm{C}$. The abovementioned methods are based on time-consuming photochemical and thermal reactions and nanoparticle precursors, which limit the initial concentration to avoid extensive aggregation. The high demand for silver sulfide nanoparticles in many applications is a clear motivation to precisely control the particles' size and shape by simple and scalable procedures. Microwave irradiation emerged as a synthesis method because of rapid and controllable volumetric heating, unique reaction effects, and increased reaction rates. Gao et al. ${ }^{12}$ have reported a onestep interface reaction of synthesis and assembly of monodispersed silver nanoparticles using the microwave irradiation approach. They found that adjusting the proportions between dodecanethiol and ethylene glycol, rectangular nanocrystals that had been arranged into orthogonal superlattices of silver nanoparticles were obtained. On the other hand, substituting ethylene glycol with an aqueous thiourea solution resulted in an assembly of $\mathrm{Ag}_{2} \mathrm{~S}$ nanoparticles. ${ }^{12}$

Following on the microwave-assisted synthesis of $\mathrm{Ag}_{2} \mathrm{~S}$, this work aimed to understand how the synthesis parameters such as the use of different molecular precursors (instead of $\mathrm{Ag}$ nanoparticles), synthesis time, and light irradiation influenced the formation of $\mathrm{Ag}_{2} \mathrm{~S}$ nanoparticles in a microwave reactor and their morphology.

\section{RESULTS AND DISCUSSION}

Influence of Ag Source. Silver sulfide nanoparticle synthesis was attempted using three different precursors: $\mathrm{AgNO}_{3}, \mathrm{Ag}_{2} \mathrm{O}$, and $\mathrm{AgF}_{2}$. The synthesis with $\mathrm{AgNO}_{3}$ and 1dodecanethiol was based on a previous report. ${ }^{10}$ Briefly, a mixture of $1 \mathrm{mmol}$ of $\mathrm{AgNO}_{3}$ and $5 \mathrm{~g}$ of 1-dodecanethiol was purged with $\mathrm{N}_{2}$ and heated in a microwave reactor to $300{ }^{\circ} \mathrm{C}$ for $10 \mathrm{~min}$. The temperature was maintained for $5 \mathrm{~min}$, and the vessel was under white light irradiation from the reactor's internal lamp. The same conditions were used for the nanoparticle synthesis from $\mathrm{Ag}_{2} \mathrm{O}$ and $\mathrm{AgF}_{2}$. Figure 1 shows a schematic of the reported synthesis.

From the chosen silver precursors, $\mathrm{AgNO}_{3}$ and $\mathrm{Ag}_{2} \mathrm{O}$ resulted in silver sulfide, as shown in the $\mathrm{X}$-ray diffraction (XRD) patterns in Figure 2A1,A2. The relatively high intensity from the silver diffraction peaks at $64.4^{\circ}$ and $77.3^{\circ}$ reveal that the synthesis with $\mathrm{AgF}_{2}$ yielded more metallic silver than silver sulfide. Figure 1 AR includes the standard patterns calculated from the data available in the Crystallography Open Database (Revision 204654-2018/01/02) using the reference 96-1100137 for $\mathrm{Ag}$ and $96-900-0254$ for $\mathrm{Ag}_{2} \mathrm{~S}$.

Both silver nitrate and silver oxide generated silver sulfide nanoparticles, with size ranges of $7 \pm 3$ and $5 \pm 2 \mathrm{~nm}$, respectively. In the TEM images of the two syntheses, a typical closely packed arrangement of silver sulfide nanoparticles is observed, which was also reported by Gao et al. ${ }^{12}$ The spherical particle shape is comparable to the nanoparticles obtained by Tian et al. ${ }^{10}$ but their synthesis was carried out in $7 \mathrm{~h}$, instead of a total synthesis time of $15 \mathrm{~min}$, as described in the present work. In a microwave-assisted synthesis, the rapid

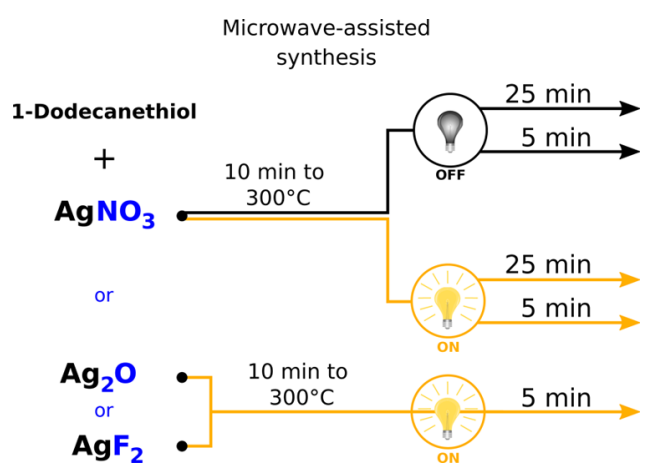

Figure 1. Schematic of all the microwave-assisted synthesis reported in this work. The mixture of 1-dodecanethiol and different precursors $\left(\mathrm{AgNO}_{3}, \mathrm{Ag}_{2} \mathrm{O}\right.$, or $\left.\mathrm{AgF}_{2}\right)$ is heated to $300{ }^{\circ} \mathrm{C}$ for $10 \mathrm{~min}$ and maintained at that temperature for a certain time. The synthesis performed with $\mathrm{AgNO}_{3}$ was either carried out in the dark or with the reactor's lamp ON.

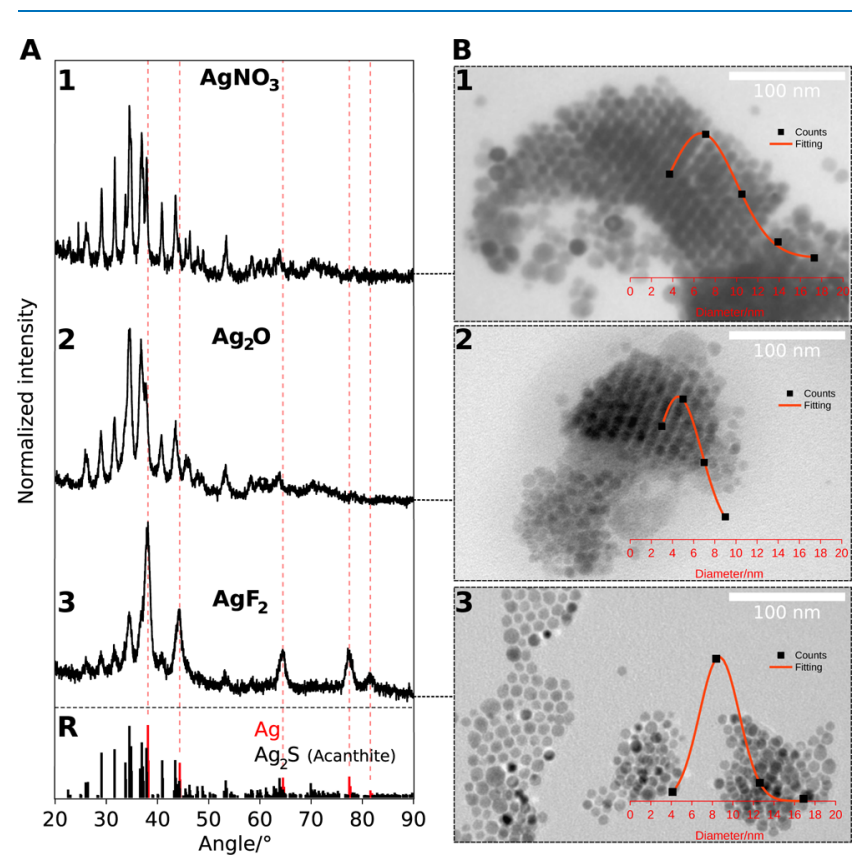

Figure 2. XRD patterns (A) and transmission electron microscopy images (TEM) (B) of the synthesized nanoparticles, starting from $\mathrm{AgNO}_{3}$ (1), $\mathrm{Ag}_{2} \mathrm{O}$ (2), and $\mathrm{AgF}_{2}$ (3). On the bottom of the XRD patterns are the standard diffraction patterns of silver and silver sulfide (acanthite) (R). In the TEM images, the estimated particle size distributions are shown.

energy supply leads to the acceleration of chemical reactions, which explains the significant improvement in synthesis time. ${ }^{13}$

The silver nanoparticles that resulted from the synthesis with $\mathrm{AgF}_{2}$ have an average size of $9 \pm 2 \mathrm{~nm}$. Silver(II) fluoride, coined as the greedy oxidizer by Grochala, ${ }^{14}$ is a strong oxidizer, and because 1-dodecanethiol is a mild reducer, we speculate that the silver(II) ion is simply reduced to metallic silver.

Silver nitrate was chosen as the precursor to proceed with the synthesis with variable time and illumination experiments because the oxide and the fluoride are less practical, more dangerous to handle, and less stable when stored under ambient conditions.

Influence of Synthesis Time and Light. The synthesis was performed keeping the conditions used in the precursor 
study by increasing the synthesis time to $25 \mathrm{~min}$ and with microwave chamber light ON or OFF. XRD and TEM results are shown in Figure 3A,B. The XRD patterns reveal that both

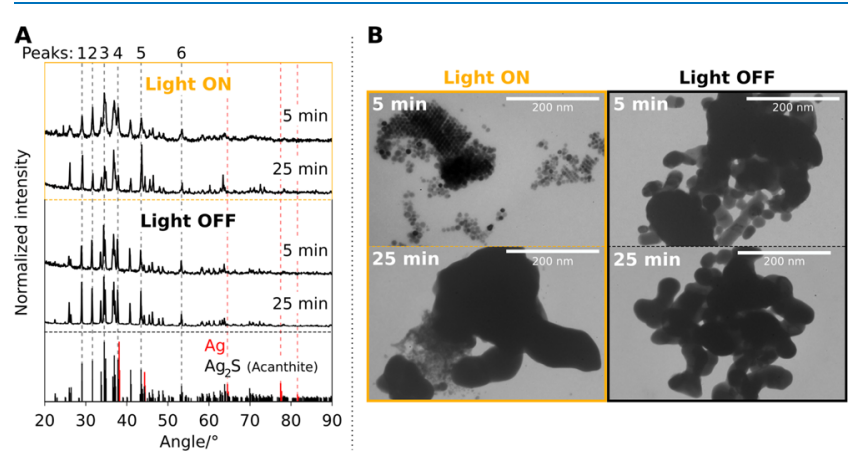

Figure 3. XRD patterns (A) and TEM images (B) of the powders resulting from synthesis with silver nitrate as the precursor, different illumination settings (light $\mathrm{ON}$ and light OFF), and synthesis time at $300{ }^{\circ} \mathrm{C}$ (5 and $25 \mathrm{~min}$ ). Standard diffraction patterns of the silver $\left(\mathrm{Ag}^{\circ}\right)$ and silver sulfide acanthite phase are shown at the bottom of (A). Peaks 1, 2, 3, 4, 5, and 6 correspond to the $\mathrm{Ag}_{2} \mathrm{~S}$ acanthite phase.

conditions lead to pure $\mathrm{Ag}_{2} \mathrm{~S}$ because $\mathrm{Ag}$ is practically absent. Taking into account that the intensities of peak 6 , as shown in Figure $3 \mathrm{~A}$, are similar between experiments, as the synthesis time increases, the particles grow along the $\operatorname{Ag}_{2} \mathrm{~S}$ (acanthite) [111] and [220] directions. This is observed at a $2 \theta$ of 29 and $44^{\circ}$ (peaks 1 and 5, respectively) because the intensities increase, compared to the $5 \mathrm{~min}$ synthesis, regardless of whether the light is $\mathrm{ON}$ or OFF. Elongation of silver sulfide nanoparticles synthesized in a microwave reactor has been described by Yaghmour and Mahmoud. ${ }^{15}$ The TEM images confirm that the particles become bigger and elongated with the increased reaction time (Figure 3B). However, the particles growth is affected by the chamber light. The intensities of peaks 2,3 , and 4, respectively, in directions [-112], [-121], and $[-103]$ decrease when the particles are synthesized for 25 min at $300^{\circ} \mathrm{C}$ with light $\mathrm{ON}$, while in the dark, it remains the same. The TEM images of the 5 min synthesis at $300{ }^{\circ} \mathrm{C}$ show small and regular $\mathrm{Ag}_{2} \mathrm{~S}$ nanoparticles when light is $\mathrm{ON}$. As synthesis at $300{ }^{\circ} \mathrm{C}$ continues, a shadowing effect caused by newly formed black silver sulfide particles can block incoming light. Hence, the bigger particles/clusters in the $25 \mathrm{~min}$ synthesis with light ON were observed, as shown in Figure 3B. Particles that were synthesized with light OFF have their length enlarged up to more than $100 \mathrm{~nm}$.

Jin et al. ${ }^{8}$ first demonstrated in 2001 a photoinduced method for synthesizing large quantities of silver nanoprisms. Their process starts with a typical synthesis of spherical nanoparticles that when irradiated with a fluorescent lamp for $70 \mathrm{~h}$, a color change in the suspension from yellow to green and then to blue is observed, indicating a shape transformation from spherical to triangular prismatic nanoparticles. Recalling that visible light promotes a photocatalytic process leading to the formation of $\mathrm{Ag}^{+}$ions at the initial stage, and the subsequent reduction promotes the growth in preferential directions, ${ }^{8}$ we propose that for the synthesis of silver sulfide under irradiation, a similar mechanism is in action. Tian et al. ${ }^{10}$ suggest that $\mathrm{Ag}$ nuclei are formed during the synthesis of $\mathrm{Ag}_{2} \mathrm{~S}$ with 1dodecanethiol, but under irradiation, the nuclei are actively digested by light-activated thiol radicals, ${ }^{11}$ and $\mathrm{Ag}^{+}$returns to the solution, hindering the particle growth.
The optical properties of the $\mathrm{Ag}_{2} \mathrm{~S}$ nanoparticles were investigated by photoluminescence (PL) at $67 \mathrm{~K}$. As shown in Figure $4 \mathrm{~A}$, the spectra for nanoparticles synthesized with
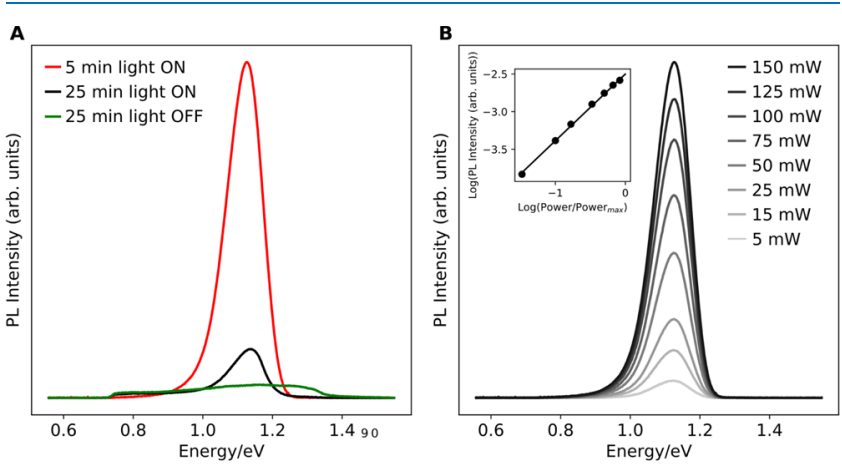

Figure 4. (A) Photoluminescence spectra of the nanoparticles synthesized with $\mathrm{AgNO}_{3}$ for 5 and 25 min with light $\mathrm{ON}$ and 25 min with light OFF, measured at $67 \mathrm{~K}$ and with an excitation power of $150 \mathrm{~mW}$. (B) Inset shows a superposition of the maximum of each PL spectrum for different excitation power values from the nanoparticles synthesized with light $\mathrm{ON}$ for $5 \mathrm{~min}$.

$\mathrm{AgNO}_{3}$ for 5 and $25 \mathrm{~min}$, with light $\mathrm{ON}$ and OFF in the chamber, were recorded. For the sample synthesized without light, a very broad band with a low intensity is observed. Such a behavior is typical of materials with high density of defects. ${ }^{16}$ In the case of nanoparticles synthesized with light $\mathrm{ON}$, a relatively broad and asymmetric band is observed in the range of $0.92-1.24 \mathrm{eV}$. Similar asymmetric behavior into the second near-infrared window (NIR-II, 0.73-1.24 eV) is observed in the literature for $\mathrm{Ag}_{2} \mathrm{~S}$ quantum dots embedded on different solutions. ${ }^{17-19}$ In particular, we note an approximate exponential decrease of the PL intensity in the low energy side, whereas for the high energy side, a steeper decrease is observed. Luminescence with these characteristics is common in materials showing a high level of disorder in which the density of states associated with the energy levels involved in radiative transitions is small. ${ }^{20,21}$ We should note that the shape of the luminescence does not depend on the excitation power, as shown in Figure 4B. For the two samples with nanoparticles synthesized with light $\mathrm{ON}$, the energy range of the observed luminescence is $\sim 0.91-1.24 \mathrm{eV}$. Thus, the band gap energy for the nanoparticles should be higher or equal to $\sim 1.24 \mathrm{eV}$. Comparing this value with the ones expected to the bulk $\mathrm{Ag}_{2} \mathrm{~S}(0.9-1.1 \mathrm{eV}){ }^{22}$ our results suggest a moderate quantum confinement effect responsible for the increase of the band gap energy. Actually, the average dimension estimated $(\sim 7 \mathrm{~nm})$ for the nanoparticles synthesized for $5 \mathrm{~min}$, is not low enough to attain the very high band gap values, as reported in the literature. ${ }^{23}$ The dependence on the excitation power $(P)$ of the luminescence was investigated (Figure 4B). With the increase of the excitation power, an apparent blueshift of the luminescence from the nanoparticles synthesized with light $\mathrm{ON}$ for $5 \mathrm{~min}$, is observed. The inset in Figure 4B shows such a dependence involving the PL integrated intensity $(I)$. $I$ was calculated through a fitting procedure to the PL spectra, as shown in Figure S2. The experimental points were fitted with the power law $P^{m},{ }^{24}$ and an estimated value of $m=0.89 \pm 0.02$ was obtained. This value is similar to the ones reported for materials where fluctuations of the electric potential are present $^{25,26}$ and can be related with the radiative recombination of charge carriers with some degree of localization 
compatible with low density of states involved in this recombination channel. This low density of states is commonly achieved when the density of defects in the lattice is high enough to create radiative states inside the band gap. Consequently, a high enough energy shift of the peak of the luminescence to the band gap energy is expected. Thus, the overall experimental behavior of the luminescence suggests the observation of just one radiative transition. The presence of high density of lattice defects in $\mathrm{Ag}_{2} \mathrm{~S}$ has been discussed in the literature and was related with the tendency of deviation from the stoichiometry of the material. ${ }^{23}$

Despite the necessarily different optical alignments in the PL measurements for these two samples, the results suggest that for a synthesis of $5 \mathrm{~min}$ with light $\mathrm{ON}$, the intensity of the luminescence is clearly higher than for $25 \mathrm{~min}$ with light $\mathrm{ON}$. Actually, the values of 1429 and 269 were obtained for the estimated signal-to-noise ratio for $5 \mathrm{~min}$ with light $\mathrm{ON}$ and 25 min with light $\mathrm{ON}$ samples, respectively. Therefore, our results show an influence of the mean size of the nanoparticles on the PL intensity. For the nanoparticles with the lower size, the relative influence of the surface is higher on the electronic energy level structure. The increase of the PL intensity for the nanoparticles with the lower size suggests that the radiative recombination channels progressively dominate over the nonradiative ones. This is compatible with a material with high density of defects in the lattice in which the surface can have a passivation role on the defects. ${ }^{19}$

A final remark regarding the reproducibility of the synthesis is stated in the Supporting Information. The color profiles plotted against synthesis time for the synthesis carried out with light $\mathrm{ON}$ for 5 and $15 \mathrm{~min}$ at $300{ }^{\circ} \mathrm{C}$ are shown, as a way to demonstrate that the transformations that occur along the synthesis are similar. For further details on the verification method, please refer to the previous report. ${ }^{27}$

\section{CONCLUSIONS}

In an attempt to control the microwave-assisted synthesis of silver sulfide nanoparticles, parameters such as the starting silver reagent, synthesis time, and illumination setting were tested.

Small nanoparticles $(6-10 \mathrm{~nm})$ of silver sulfide were obtained when silver(I) nitrate and silver(I) oxide were used, but when the reagent was switched to silver(II) fluoride, metallic silver was obtained predominantly. Regarding the influence of the synthesis time and irradiation during synthesis, it was first demonstrated that the geometry and size of silver sulfide nanoparticles are affected. Small silver sulfide nanoparticles were obtained when the synthesis time was shorter (5 min) and under irradiation ( $7 \mathrm{~nm}$ mean size). Rods with a length of tens of nanometers were obtained when the synthesis was carried out in the dark for a longer time $(25 \mathrm{~min})$. A clear influence of light on the recombination channels of photogenerated charge carriers on the nanoparticles was observed. The synthesis performed without light resulted in the almost absence of luminescence, whereas for the synthesis performed under light, the radiative recombination of carriers is typical of materials characterized by the involvement of low density of states inside the band gap.

\section{MATERIALS AND METHODS}

Silver(I) nitrate ( $\geq 99.8 \%)$, silver(II) fluoride ( $\geq 98 \%)$, and 1dodecanethiol $(\geq 98 \%)$ were purchased from Sigma-Aldrich.
Silver(I) oxide (99\%, metal basis) was purchased from Alfa Aesar. Ethanol absolute anhydrous (ACS-Reag., Ph.Eur.-Reag. USP) was purchased from CARLO ERBA Reagents. Synthesis of silver sulfide particles was achieved by mixing $1 \mathrm{mmol}$ of the silver precursor with $5 \mathrm{~g}$ of 1-dodecanethiol, purging with a constant flow of nitrogen gas for $15 \mathrm{~min}$, under rapid stirring with a magnet, and heating in a microwave reactor to $300{ }^{\circ} \mathrm{C}$ for $10 \mathrm{~min}$. The temperature of $300{ }^{\circ} \mathrm{C}$ was maintained for 5 min for all silver precursors and for $25 \mathrm{~min}$ for silver nitrate. The microwave reactor internal lamp was kept on for all silver precursors and off for silver nitrate. The light spectra recorded, using a Sarspec SPEC SENSE + UV/VIS/NIR from the lamp, can be found in the Supporting Information in Figure S2. After the set time, the mixture was cooled down to $50{ }^{\circ} \mathrm{C}$ with compressed air. The suspension was collected and centrifuged, and the excess dodecanethiol was decanted. The powder was resuspended in ethanol, vigorously agitated with the aid of a vortex mixer, and centrifuged three more times. The synthesis was performed in an Anton Paar Microwave Synthesis Reactor Monowave 400, and images were recorded using the reactor's integrated camera.

The nanoparticle structure was confirmed by XRD in an X'Pert PRO MPD, with a Cu K- $\alpha$ source at the wavelength of 1.540598 Å. TEM images were obtained from a Hitachi $\mathrm{H}$ 8100 II after depositing an ethanolic suspension of the washed nanoparticles on copper grids.

The optical characterization was performed through PL measurements with a Bruker Vertex $80 \mathrm{v}$ Fourier transform infrared spectrometer equipped with a Hypherion microscope unit and using as the excitation source a laser with the wavelength of $532 \mathrm{~nm}$. The laser power was measured at the front of the spectrometer window. The excitation power was varied from 5 to $150 \mathrm{~mW}$, and the temperature was fixed at 67 $\mathrm{K}$ for all samples.

\section{ASSOCIATED CONTENT}

\section{SI Supporting Information}

The Supporting Information is available free of charge at https://pubs.acs.org/doi/10.1021/acsomega.0c00656.

Reproducibility of synthesis, microwave reactor lamp's light spectrum, PL spectra at various laser powers, and corresponding fittings (PDF)

\section{AUTHOR INFORMATION}

\section{Corresponding Author}

David Magalhães Sousa - CENIMAT/I3N, Departamento de Cîncia dos Materiais, Universidade de Aveiro, 3810-193 Aveiro, Portugal; 이이. orcid.org/0000-0002-9810-2798; Email:dma.sousa@campus.fct.unl.pt
Authors
William Chiappim - i3N, Departamento de Física, Universidade de Aveiro, 3810-193 Aveiro, Portugal
Joaquim P. Leitão - i3N, Departamento de Fisica, Universidade de Aveiro, 3810-193 Aveiro, Portugal; ㅇo orcid.org/0000- 0001-8131-3313
João Carlos Lima - LAQV-REQUIMTE, Departamento de Quimica, CQF B. Faculdade de Ciencias e Tecnologia, Universidade NOVA de Lisboa, 2829-516 Caparica, Portugal; ○ orcid.org/0000-0003-0528-1967 
Isabel Ferreira - CENIMAT/I3N, Departamento de Cîncia dos Materiais, Universidade de Aveiro, 3810-193 Aveiro, Portugal; (1) orcid.org/0000-0002-8838-0364

Complete contact information is available at:

https://pubs.acs.org/10.1021/acsomega.0c00656

\section{Notes}

The authors declare no competing financial interest.

\section{ACKNOWLEDGMENTS}

This work was mainly funded by the ERC-CoG-2014, ChapTherPV, 647596 and partially by the H2020-ICT-20141, RIA, TransFlexTeg, 645241, SusPhotoSolutions, i3N, UIDB/50025/2020, UIDP/50025/2020, UID/CTM/50025/ 2013, and UID/CTM/50025/2019 and FEDER funds through the COMPETE 2020 Program and National Funds through FCT-Portuguese Foundation for Science and Technology.

\section{REFERENCES}

(1) Li, L.; Zhu, B.; Yan, X.; Zhou, Q.; Wang, Y.; Jiang, G. Effect of Silver Sulfide Nanoparticles on Photochemical Degradation of Dissolved Organic Matter in Surface Water. Chemosphere 2018, 193, 1113-1119.

(2) Soriano-Molina, P.; Plaza-Bolaños, P.; Lorenzo, A.; Agüera, A.; García Sánchez, J. L.; Malato, S.; Sánchez Pérez, J. A. Assessment of Solar Raceway Pond Reactors for Removal of Contaminants of Emerging Concern by Photo-Fenton at Circumneutral PH from Very Different Municipal Wastewater Effluents. Chem. Eng. J. 2019, 366, 141-149.

(3) Li, L.; Wang, Y.; Liu, Q.; Jiang, G. Rethinking Stability of Silver Sulfide Nanoparticles (Ag 2 S-NPs) in the Aquatic Environment: Photoinduced Transformation of Ag 2 S-NPs in the Presence of $\mathrm{Fe}(\mathrm{III})$. Environ. Sci. Technol. 2016, 50, 188-196.

(4) Li, L.; Zhou, Q.; Geng, F.; Wang, Y.; Jiang, G. Formation of Nanosilver from Silver Sulfide Nanoparticles in Natural Waters by Photoinduced Fe(II, III) Redox Cycling. Environ. Sci. Technol. 2016, 50, 13342-13350.

(5) Kamat, P. V.; Flumiani, M.; Hartland, G. V. Picosecond Dynamics of Silver Nanoclusters. Photoejection of Electrons and Fragmentation. J. Phys. Chem. B 1998, 102, 3123-3128.

(6) Procházka, M. Applications of Laser-Ablated Ag Colloids in SERS Spectroscopy: Improvement of Ablation Procedure and SERS Spectral Testing. Anal. Chem. 1997, 69, 5103-5108.

(7) Henglein, A. Reduction of $\operatorname{Ag}(\mathrm{CN}) 2$ - on Silver and Platinum Colloidal Nanoparticles. Langmuir 2001, 17, 2329-2333.

(8) Jin, R.; Cao, Y.; Mirkin, C. A.; Kelly, K. L.; Schatz, G. C.; Zheng, J. G. Photoinduced Conversion of Silver Nanospheres to Nanoprisms. Science 2001, 294, 1901-1903.

(9) Xue, C.; Métraux, G. S.; Millstone, J. E.; Mirkin, C. A. Mechanistic Study of Photomediated Triangular Silver Nanoprism Growth. J. Am. Chem. Soc. 2008, 130, 8337-8344.

(10) Tian, C.; Kang, Z.; Wang, E.; Mao, B.; Li, S.; Su, Z.; Xu, L. 'One-Step' Controllable Synthesis of Ag and Ag2S Nanocrystals on a Large Scale. Nanotechnology 2006, 17, 5681-5685.

(11) Cao, Y.; Geng, W.; Shi, R.; Shang, L.; Waterhouse, G. I. N.; Liu, L.; Wu, L.-Z.; Tung, C.-H.; Yin, Y.; Zhang, T. Thiolate-Mediated Photoinduced Synthesis of Ultrafine Ag2S Quantum Dots from Silver Nanoparticles. Angew. Chem., Int. Ed. 2016, 55, 14952-14957.

(12) Gao, F.; Lu, Q.; Komarneni, S. Interface Reaction for the SelfAssembly of Silver Nanocrystals under Microwave-Assisted Solvothermal Conditions. Chem. Mater. 2005, 17, 856-860.

(13) Zhu, Y.-J.; Chen, F. Microwave-Assisted Preparation of Inorganic Nanostructures in Liquid Phase. Chem. Rev. 2014, 114, 6462-6555.

(14) Grochala, W. Greedy $\mathrm{Ag}(\mathrm{II})$ Oxidizer: Can Any Inorganic Ligand except Fluoride Endure Its Presence in Ionic Solids? J. Fluor. Chem. 2008, 129, 82-90.
(15) Yaghmour, S. J.; Mahmoud, W. E. Synthesis and Characterization of Self-Assembly Silver Sulfide Nanorods Prepared by Squalene Assisted Microwave Technique. Mater. Lett. 2013, 109, $55-57$.

(16) Santos, N. M.; Falcão, B. P.; Leitão, J. P.; Sobolev, N. A.; Carmo, M. C.; Stepina, N. S.; Yakimov, A.; Nikiforov, A. I. Optical Study of Strained Double Ge/Si Quantum Dot Layers. IOP Conf. Ser.: Mater. Sci. Eng. 2009, 6, 012018.

(17) Theodorou, I. G.; Jawad, Z. A. R.; Qin, H.; Aboagye, E. O.; Porter, A. E.; Ryan, M. P.; Xie, F. Significant Metal Enhanced Fluorescence of Ag $2 \mathrm{~S}$ Quantum Dots in the Second Near-Infrared Window. Nanoscale 2016, 8, 12869-12873.

(18) Theodorou, I. G.; Jiang, Q.; Malms, L.; Xie, X.; Coombes, R. C.; Aboagye, E. O.; Porter, A. E.; Ryan, M. P.; Xie, F. Fluorescence Enhancement from Single Gold Nanostars: Towards Ultra-Bright Emission in the First and Second near-Infrared Biological Windows. Nanoscale 2018, 10, 15854-15864.

(19) Ovchinnikov, O. V.; Grevtseva, I. G.; Smirnov, M. S.; Kondratenko, T. S. Reverse Photodegradation of Infrared Luminescence of Colloidal Ag2S Quantum Dots. J. Lumin. 2019, 207, 626632.

(20) Levanyuk, A. P.; Osipov, V. V. Edge Luminescence of DirectGap Semiconductors. Sov. Phys. Usp. 1981, 24, 187-215.

(21) Teixeira, J. P.; Sousa, R. A.; Sousa, M. G.; da Cunha, A. F.; Fernandes, P. A.; Salomé, P. M. P.; Leitão, J. P. Radiative Transitions in Highly Doped and Compensated Chalcopyrites and Kesterites: The Case of Cu2ZnSnS4. Phys. Rev. B 2014, 90, 235202.

(22) Zamiri, R.; Abbastabar Ahangar, H.; Zakaria, A.; Zamiri, G.; Shabani, M.; Singh, B.; Ferreira, J. M. F. The Structural and Optical Constants of Ag2S Semiconductor Nanostructure in the Far-Infrared. Chem. Cent. J. 2015, 9, 28.

(23) Ovchinnikov, O. V.; Smirnov, M. S.; Shapiro, B. I.; Shatskikh, T. S.; Perepelitsa, A. S.; Korolev, N. V. Optical and Structural Properties of Ensembles of Colloidal Ag2S Quantum Dots in Gelatin. Semiconductors 2015, 49, 373-379.

(24) Schmidt, T.; Lischka, K.; Zulehner, W. Excitation-Power Dependence of the near-Band-Edge Photoluminescence of Semiconductors. Phys. Rev. B 1992, 45, 8989-8994.

(25) Salome, P. M. P.; Teixeira, J. P.; Keller, J.; Torndahl, T.; Sadewasser, S.; Leitao, J. P. Influence of $\mathrm{CdS}$ and $\mathrm{ZnSnO}$ Buffer Layers on the Photoluminescence of $\mathrm{Cu}(\mathrm{In}, \mathrm{Ga}) \mathrm{Se} \$ 2 \$$ Thin Films. IEEE J. Photovoltaics 2017, 7, 670-675.

(26) Ben Sedrine, N.; Ribeiro-Andrade, R.; Gustafsson, A.; Soares, M. R.; Bourgard, J.; Teixeira, J. P.; Salomé, P. M. P.; Correia, M. R.; Moreira, M. V. B.; De Oliveira, A. G.; González, J. C.; Leitão, J. P. Fluctuating Potentials in GaAs:Si Nanowires: Critical Reduction of the Influence of Polytypism on the Electronic Structure. Nanoscale 2018, 10, 3697-3708.

(27) Sousa, D. M.; Alves, L. C.; Marques, A.; Gaspar, G.; Lima, J. C.; Ferreira, I. Facile Microwave-Assisted Synthesis Manganese Doped Zinc Sulfide Nanoparticles. Sci. Rep. 2018, 8, 15992. 